\title{
Diagnosis and conservative treatment of a rare case of femoral intraosseous arteriovenous malformation in a patient with polyostotic fibrous dysplasia: A case report
}

\author{
SHUSA OHSHIKA $^{1}$, MICHIRO YANAGISAWA ${ }^{1}$, FUMIYASU TSUSHIMA ${ }^{2}$ and YASUYUKI ISHIBASHI ${ }^{1}$ \\ Departments of ${ }^{1}$ Orthopaedic Surgery and ${ }^{2}$ Radiology, Hirosaki University Graduate School of Medicine, \\ Hirosaki, Aomori 036-8562, Japan
}

Received January 31, 2019; Accepted April 5, 2019

DOI: $10.3892 / \mathrm{mco} .2019 .1845$

\begin{abstract}
Pure intraosseous arteriovenous malformation (AVM) in a limb bone is extremely rare. Furthermore, there is currently insufficient information on the diagnostic and therapeutic strategies for pure intraosseous AVMs. We herein report a case of pure intraosseous AVM of the proximal femur occurring in a patient with polyostotic fibrous dysplasia. The patient was a 39-year-old woman who presented with pain in the right thigh. Plain radiographs and computed tomography scans revealed a medullary lytic lesion with expansion and thinning of the bone cortex in the right proximal femur, mimicking a primary bone tumor. Magnetic resonance imaging (MRI) examination revealed intramedullary signal voids and feeding arteries arising from the deep femoral artery. A non-surgical approach using embolization and denosumab achieved satisfactory results, which included complete obliteration of the AVM, increased cortical thickness of the right proximal femur, and attenuation of the high-turnover bone metabolism 1 year later. Careful review of MRI images is crucial for distinguishing between bone tumors and intraosseous AVM, which exhibit signal voids and feeding arteries, in order to avoid unnecessary interventions such as bone biopsy or surgery.
\end{abstract}

\section{Introduction}

The International Society for the Study of Vascular Anomalies (ISSVA) classifies vascular anomalies into vascular tumors and

Correspondence to: Dr Shusa Ohshika, Department of Orthopaedic Surgery, Hirosaki University Graduate School of Medicine, 5 Zaifu-cho, Hirosaki, Aomori 036-8562, Japan

E-mail: ohshika@hirosaki-u.ac.jp

Abbreviations: AVM, arteriovenous malformation; poly-FD, polyostotic fibrous dysplasia

Key words: intraosseous arteriovenous malformation, polyostotic fibrous dysplasia, signal void, denosumab, embolization vascular malformations, according to the biological classification proposed by Mulliken and Glowacki $(1,2)$. Arteriovenous malformations (AVMs) are high-flow vascular malformations in which well-formed arterial and venous elements communicate directly, rather than through a normal capillary network. Although the majority of AVMs occur in soft tissues, osseous involvement is occasionally encountered, with symptoms such as pain, mass effects with pulsation, swelling of the extremity, skin ulceration, bone overgrowth, bone destruction, and heart failure $(3,4)$.

Intraosseous AVMs, which are relatively rare, predominantly occur in craniofacial bones, such as the mandible and maxilla (5,6). Pure intraosseous AVMs that do not involve soft tissue are extremely rare $(3,4)$. Although several intraosseous AVMs have been described in the spine or long bones (7-14), little is known on the diagnostic and therapeutic strategies for pure intraosseous AVMs. It is crucial for physicians, particularly orthopedic oncologists, to understand the clinical characteristics of pure intraosseous AVMs in order to distinguish them from primary or metastatic bone tumors. We herein present a case of pure intraosseous AVM of the proximal femur in a patient with polyostotic fibrous dysplasia, which was treated by embolization and denosumab, and discuss the diagnostic characteristics and therapeutic approach.

\section{Case report}

A 37-year-old woman was referred to the Hirosaki University Hospital (Hirosaki, Japan) on December, 2014 with a 1-year history of pain in the left thigh while walking. The patient's medical history included a diagnosis of polyostotic fibrous dysplasia (poly-FD) when she was in her 20s at another hospital, without endocrinopathy or skin pigmentation. Plain radiographs and computed tomography (CT) examination revealed a central, longitudinal, medullary lytic lesion of the left proximal femur, with expansion and thinning of the bone cortex (Fig. 1A and B). An intramedullary lytic lesion and endosteal scalloping were also observed in the right proximal femur. There were multiple intramedullary lesions with a ground-glass appearance and lytic lesions throughout the bilateral femora and pelvis. Magnetic resonance imaging (MRI) revealed a lesion in the left proximal femur that exhibited a 
homogeneous intermediate signal on T1-weighted images and hyperintensity on the T2-weighted images (Fig. 1C and D). In the right proximal femur, an intramedullary hypointense lesion was identified on both T1- and T2-weighted images. An impending fracture of the left femur was diagnosed, resulting from a secondary cyst formation of the FD, and intramedullary nailing of the left femur was performed. The patient had an uneventful recovery after surgery. At the 1-year follow-up on December, 2015, she remained asymptomatic, without progressive changes in the right lower extremity on plain radiographs, and was scheduled for routine orthopedic follow-up at another hospital.

The patient visited our hospital again at the age of 39 years on November, 2016, with a 5-month history of right thigh pain while walking. Plain radiographs and CT scans revealed worsening of the findings compared with those 2 years earlier, consisting of a central, longitudinal medullary lytic lesion with expansion and thinning of the bone cortex (Fig. 2A-C). There was a mild disruption of the intertrochanteric posterior bone cortex (Fig. 2D), but there were no soft tissue masses or periosteal reaction. MRI revealed an extended intramedullary hypointense lesion on both T1- and T2-weighted images (Fig. 3A and B). The fat-suppressed post-contrast T1-weighted MRI revealed heterogeneous enhancement around the intramedullary hypointense lesion (Fig. 3C). There were no soft tissue masses near the right proximal femur. An impending fracture of the right femur was diagnosed, resulting from progression of the FD. Intramedullary nailing of the right femur was attempted, but the operation was discontinued due to rapid profuse bleeding after drilling the greater trochanter. The hemorrhage was controlled by filling the drill hole with bone wax. A review of the preoperative MRI revealed several feeding arteries arising from the deep femoral artery that were responsible for the intramedullary hypointense signal (signal void; Fig. 3D and E). Contrast-enhanced CT and 3-dimensional CT images obtained on the day after the discontinued surgery revealed marked intramedullary enhancement of the proximal femoral lesion and multiple hypertrophied perforating arteries arising from the deep femoral artery (Fig. 4A-C). Selective angiography of the deep femoral artery demonstrated multiple hypertrophied perforating arteries penetrating the femoral cortex, as well as early formation of the nidus and venous sac within the medullary cavity of the femur (Fig. 4D and E). These findings indicated that the proximal femoral lesion was an intraosseous AVM.

Therapeutic embolization was then performed for a total of 3 times (on days 2, 9 and 21 after the initial surgery). In the first and second embolizations, transarterial n-butyl-2cyanoacrylate (NBCA) injections were performed. In the third embolization, a small venous sac remaining within the medullary cavity was completely obliterated by transvenous coil embolization (Fig. 4F and G). The patient was able to stand and walk with full weight-bearing without pain on the day after the final embolization, but she was advised to walk with crutches with load restriction on the right lower extremity and to avoid activities that would increase the risk of pathological fracture for 3 months after the embolization. In addition, biochemical measurements, including serum total alkaline phosphatase (ALP), bone alkaline phosphatase (BAP), calcium, phosphate and urine $\mathrm{N}$-terminal
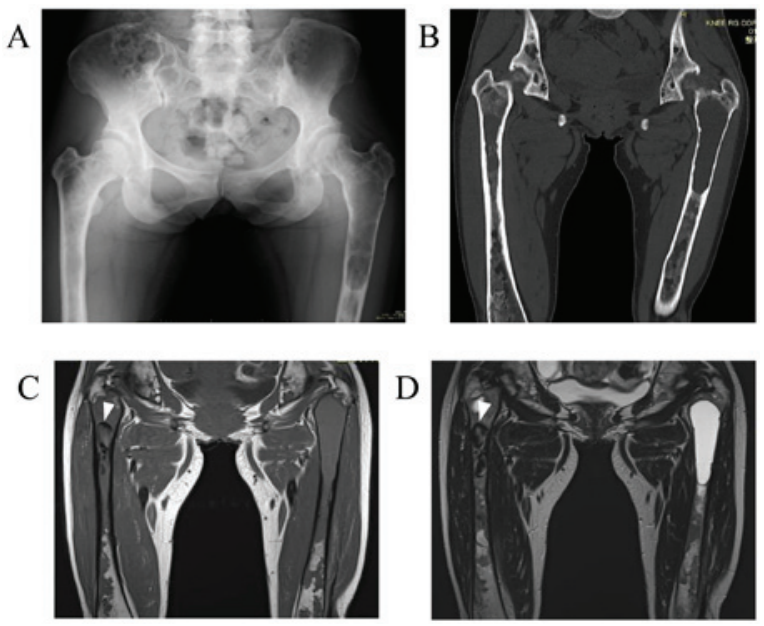

Figure 1. Initial imaging findings in a 37-year-old patient with polyostotic fibrous dysplasia. (A) Plain radiograph (anteroposterior view) and (B) coronal plain CT revealed a central, longitudinal, medullary lytic lesion in the left proximal femur with expansion and thinning of the bone cortex. In the right proximal femur, an intramedullary lytic lesion and endosteal scalloping were also observed. Coronal MRI revealed a lesion with a (C) homogeneous intermediate signal on T1-weighted images and (D) a hyperintense signal on T2-weighted images in the left proximal femur. In the right proximal femur, an intramedullary hypointense lesion (arrowheads) was observed on both T1- and T2-weighted images. CT, computed tomography; MRI, magnetic resonance imaging.
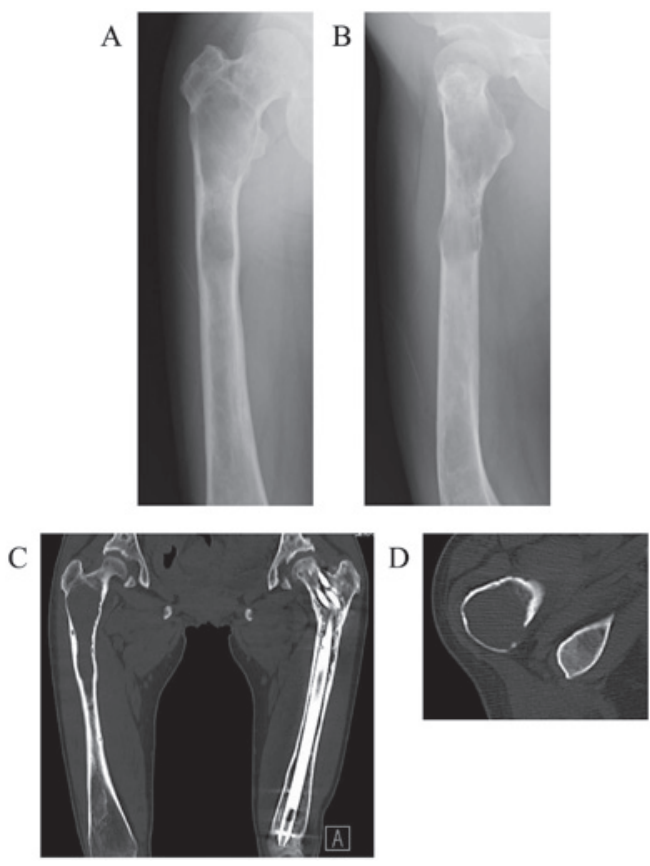

Figure 2. Imaging findings when the same patient presented again 2 years later with a 5-month history of right thigh pain. Plain radiographs of the (A) anteroposterior and (B) lateral view and (C) plain coronal computed tomography $(\mathrm{CT})$ revealed marked worsening in the changes of the right proximal femur compared with those from 2 years earlier, consisting of a central longitudinally medullary lytic lesion with expansion and thinning of the bone cortex. (D) Plain axial CT revealed slight disruption of the intertrochanteric posterior bone cortex, but no soft tissue mass or periosteal reaction were identified.

cross-linked telopeptide of type I collagen (NTX), indicated high bone turnover rate (Table I). Therefore, subcutaneous injections of $60 \mathrm{mg}$ denosumab (Pralia ${ }^{\circledR}$; once 

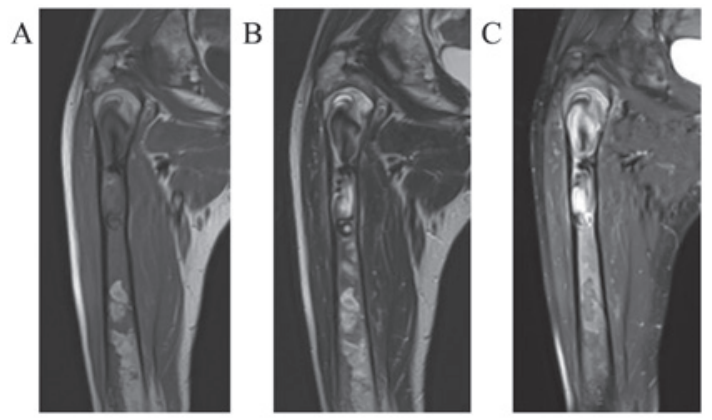

$\mathrm{D}$

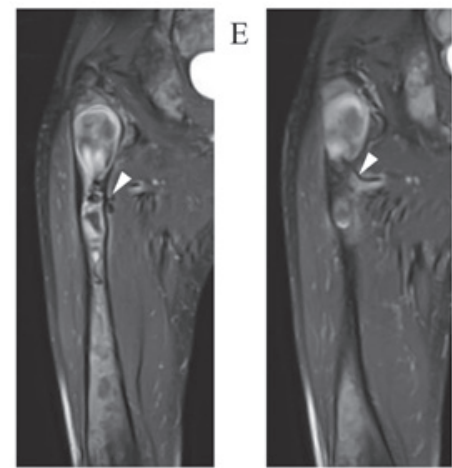

Figure 3. Magnetic resonance imaging (MRI) findings of the patient a the same time as Fig. 2. Coronal MRI revealed an extended intramedullary hypointense lesion both on (A) T1- and (B) T2-weighted images. (C-E) Coronal fat-suppressed post-contrast T1-weighted MRI revealed heterogeneous enhancement around the intramedullary hypointense lesion, as well as several feeding arteries (arrowheads) arising from the deep femoral artery.

every 6 months) with oral activated vitamin D and calcium were initiated. One year after the embolization, the patient remained asymptomatic, and the right proximal femur exhibited increased cortical thickness on plain radiographs and CT images (Fig. 5A-D). No intramedullary signal void was detected on MRI (Fig. 5E-G). Biochemical tests before and after treatment revealed attenuation of the high-turnover bone metabolism (Table I). The bone mineral density (BMD) of the lumbar spine (L2-L4) on dual-energy-X-ray absorptiometry was also increased on December, 2017 after 1 year treatment.

\section{Discussion}

The present case demonstrated that careful imaging review is crucial for distinguishing bone tumors from intraosseous AVMs, which are characterized by signal void and the presence of feeding arteries on MRI. Denosumab treatment also achieved a satisfactory result, including increased cortical thickness of the right proximal femur and attenuation of high-turnover bone metabolism. To the best of our knowledge, this is the first report of pure intraosseous AVM occurring in combination with poly-FD.

MRI examination in this case clearly demonstrated intramedullary signal voids and feeding arteries in a pure intraosseous AVM of a limb bone. MRI is an extremely important modality for assessing vascular tumors and malformations, and the presence of signal void is a hallmark MRI finding in AVMs (15-17). In previous reports (7-14), intramedullary lesions exclusively displayed a hypointense or
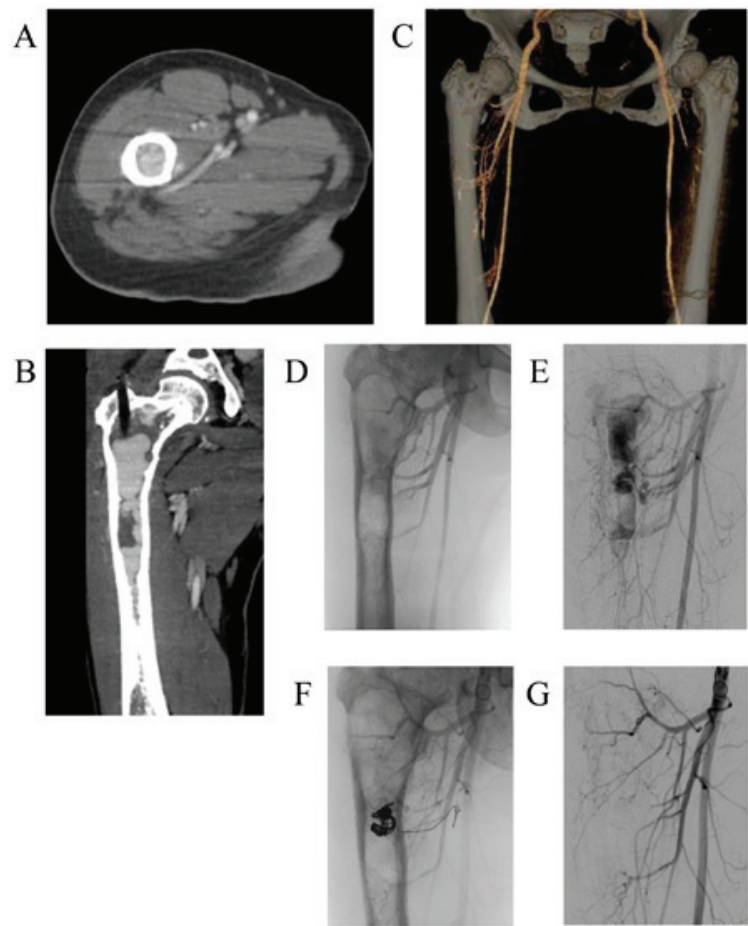

Figure 4. Contrast-enhanced computed tomography (CT) and selective angiography of the patient in Figs. 2 and 3 after discontinued surgery for intramedullary nailing of the right femur. Post-contrast (A) axial and (B) coronal CT and (C) 3-dimensional CT images revealed marked intramedullary enhancement of the right proximal femoral lesion, as well as multiple hypertrophied perforating arteries arising from the deep femoral artery. (D and E) Digital subtraction angiography images revealed multiple hypertrophied perforating arteries penetrating through the femoral cortex and early formation of the nidus and venous sac within the medullary cavity of the femur. (F and G) Digital subtraction images after the third embolization demonstrated that the venous sac had been completely obliterated by the transvenous coil embolization.

isointense signal on T1-weighted images and a hyperintense signal on T2-weighted images, without signal voids. However, in the present case, MRI demonstrated intramedullary signal voids and feeding arteries, indicating a high-flow malformation. Although the signal voids were misinterpreted as solid fibrous tissues on initial diagnosis, contrast-enhanced CT pointed towards the correct diagnosis, as it revealed marked intramedullary enhancement and hypertrophied surrounding arteries. Therefore, careful imaging review is warranted to avoid unnecessary bone biopsies and surgeries that may cause excessive bleeding.

In this case, complete obliteration of the intraosseous AVM was achieved by transarterial NBCA injections and transvenous coil embolization. The management of patients with AVM includes conservative treatment, transcatheter embolization, sclerotherapy, surgical resection, or a combination of these methods. Conservative treatment is recommended for asymptomatic or mildly impaired patients, as incomplete treatment may stimulate more aggressive growth (17). Transcatheter embolization is recommended for symptomatic, impaired patients with extensive high-flow lesions, either preoperatively or as a first-line therapeutic strategy, when surgical resection carries a significant risk of postoperative morbidity $(5,6)$. In addition, sclerotherapy may be applied preoperatively or as a definitive treatment in selected patients (18). Do et al reported 

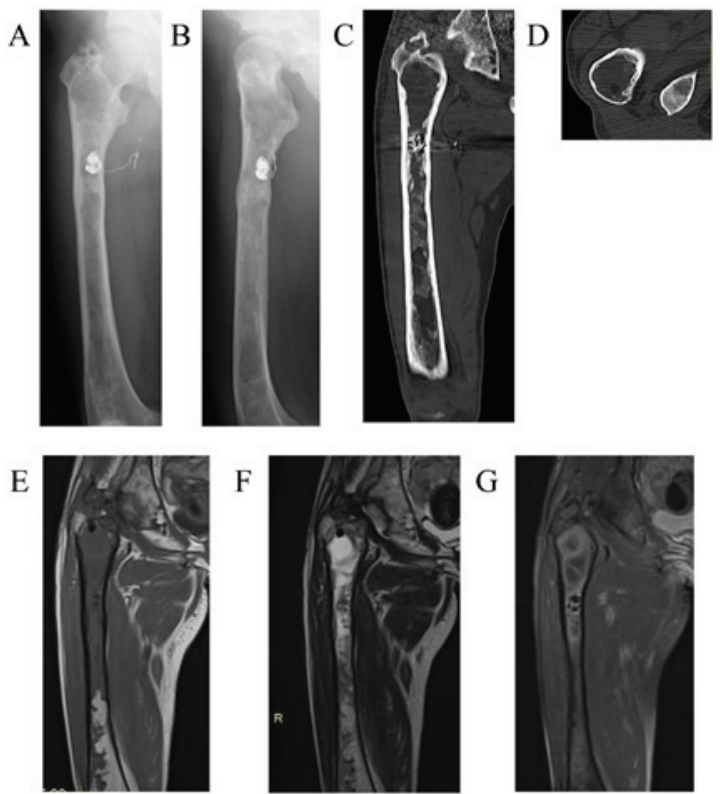

Figure 5. Imaging findings of the patient 1 year after embolization and denosumab treatment. Plain radiographs on (A) anteroposterior and (B) lateral view, as well as plain (C) coronal and (D) axial CT, revealed increased cortical thickness of the right proximal femur. Coronal MRI did not show an intramedullary signal void on (E) T1-, (F) T2-, or (G) fat-suppressed post-contrast T1-weighted images. CT, computed tomography; MRI, magnetic resonance imaging.

good results of ethanol embolotherapy for extremity AVM involving bone (3). Complete surgical resection is recommended when the AVM is small and located in a surgically accessible area.

In the present case, denosumab achieved increased cortical thickness of the proximal femur and attenuated the high-turnover bone metabolism. Pathological fracture was a concern in this case, due to the low blood supply post-embolization and the high-turnover bone metabolism. Although surgical treatment is usually recommended for FD of the lower limbs causing pain and deformity (19), good results were achieved with denosumab, which rapidly decreased the serum ALP, BAP and urine NTX levels (Table I). Several previous studies reported that intravenous pamidoronate or oral alendronate markedly relieved bone pain, improved radiological findings, increased bone density and decreased bone turnover in children or adults with poly-FD $(20,21)$. Ganda and Seibel reported that denosumab induced a rapid biochemical response and alleviated bone pain in two poly-FD patients in whom the efficacy of prior long-term bisphosphonate therapy was unsatisfactory (22). In the present case, we hypothesized that the increased cortical thickness of the proximal femur 1 year after embolization was the result of improved bone metabolism by denosumab, as well as of the spontaneous bone remodeling after the complete obliteration of the AVM.

In conclusion, we herein report a case of pure intraosseous AVM of the proximal femur in a patient with poly-FD. Non-surgical treatment by embolization and denosumab achieved a satisfactory result, which included complete obliteration of the AVM, increased cortical thickness of the proximal femur, and improvement of the high-turnover bone metabolism 1 year after embolization. Careful imaging 
review is crucial for distinguishing between bone tumors and intraosseous AVMs, which is characterized by the presence of signal voids and feeding arteries on MRI, in order to avoid unnecessary bone biopsy or surgery.

\section{Acknowledgements}

Not applicable.

\section{Funding}

No funding was received.

\section{Availability of data and materials}

All the data analyzed in this case are available from the corresponding author on reasonable request.

\section{Authors' contributions}

SO, MY, YI participated in the treatment of the patient including pharmacotherapy and surgery. FT performed arterial embolization on the patient. All the authors have read and approved the final version of this manuscript.

\section{Ethics approval and consent to participate}

Not applicable.

\section{Patient consent for publication}

We received patient consent for the publication of the case details and any associated laboratory data and images.

\section{Competing interests}

The authors declare that they have no competing interests.

\section{References}

1. Mulliken JB and Glowacki J: Hemangiomas and vascular malformations in infants and children: A classification based on endothelial characteristics. Plast Reconstr Surg 69: 412-422, 1982.

2. Enjolras O: Classification and management of the various superficial vascular anomalies: Hemangiomas and vascular malformations. J Dermatol 24: 701-710, 1997.

3. Do YS, Park KB, Park HS, Cho SK, Shin SW, Moon JW, Kim DI, Kim YW, Chang IS, Lee SH, et al: Extremity arteriovenous malformations involving the bone: Therapeutic outcomes of ethanol embolotherapy. J Vasc Interv Radiol 21: 807-816, 2010.

4. Do YS and Park KB: Special consideration for intraosseous arteriovenous malformation. Semin Intervent Radiol 34: 272-279, 2017.

5. Colletti G, Frigerio A, Giovanditto F, Biglioli F, Chiapasco M and Grimmer JF: Surgical treatment of vascular malformations of the facial bones. J Oral Maxillofac Surg 72: 1326.e1-18, 2014.
6. Theologie-Lygidakis N, Schoinohoriti O, Tzermpos F, Christopoulos P and Iatrou I: Management of intraosseous vascular malformations of the jaws in children and adolescents: Report of 6 cases and literature review. J Oral Maxillofac Res 6: e5, 2015.

7. Katzen BT and Said S: Arteriovenous malformation of bone: An experience with therapeutic embolization. AJR Am J Roentgenol 136: 427-429, 1981.

8. Nancarrow PA, Lock JE and Fellows KE: Embolization of an intraosseous arteriovenous malformation. AJR Am J Roentgenol 146: 785-786, 1986.

9. Savader SJ, Savader BL and Otero RR: Intraosseous arteriovenous malformations mimicking malignant disease. Acta Radiol 29: 109-114, 1988.

10. Knych SA, Goldberg MJ and Wolfe HJ: Intraosseous arteriovenous malformation in a pediatric patient. Clin Orthop Relat Res 276: 307-312, 1992.

11. Molina A, Martin C, Muñoz I, Aguilar L, Serrano S and Ballester J: Spinal intraosseous arteriovenous malformation as a cause of juvenile scoliosis. A case report. Spine (Phila Pa 1976) 22: 221-224, 1997

12. Matsuyama A, Aoki T, Hisaoka M, Yokoyama K and Hashimoto $\mathrm{H}$ : A case of intraosseous arteriovenous malformation with unusual radiological presentation of low blood flow. Pathol Res Pract 204: 423-426, 2008.

13. Louis RG Jr, Yen CP, Mohila CA, Mandell JW and Sheehan J: A rare intraosseous arteriovenous malformation of the spine. J Neurosurg Spine 15: 336-339, 2011.

14. Wang HH, Yeh TT, Lin YC and Huang GS: Imaging features of an intraosseous arteriovenous malformation in the tibia. Singapore Med J 56: e21-e25, 2015.

15. Fayad LM, Hazirolan T, Bluemke D and Mitchell S: Vascular malformations in the extremities: Emphasis on MR imaging features that guide treatment options. Skeletal Radiol 35: 127-137, 2006.

16. Dubois $\mathrm{J}$ and Alison M: Vascular anomalies: What a radiologist needs to know. Pediatr Radiol 40: 895-905, 2010.

17. Flors L, Leiva-Salinas C, Maged IM, Norton PT, Matsumoto AH, Angle JF, Hugo Bonatti M, Park AW, Ahmad EA, Bozlar U, et al: MR imaging of soft-tissue vascular malformations: Diagnosis, classification, and therapy follow-up. Radiographics 31: 1321-1341, 2011.

18. Kaji N, Kurita M, Ozaki M, Takushima A, Harii K, Narushima M and Wakita S: Experience of sclerotherapy and embolosclerotherapy using ethanolamine oleate for vascular malformations of the head and neck. Scand J Plast Reconstr Surg Hand Surg 43: 126-136, 2009.

19. Zhang X, Shang X, Wang Y, He R and Shi G: Intramedullary nailing for fibrous dysplasia of lower limbs. Oncol Lett 4: 524-528, 2012.

20. Chapurlat RD, Hugueny P, Delmas PD and Meunier PJ: Treatment of fibrous dysplasia of bone with intravenous pamidronate: Long-term effectiveness and evaluation of predictors of response to treatment. Bone 35: 235-242, 2004.

21. Li GD, Ogose A, Hotta T, Kawashima H, Ariizumi T, Xu Y and Endo N: Long-term efficacy of oral alendronate therapy in an elderly patient with polyostotic fibrous dysplasia: A case report. Oncol Lett 2: 1239-1242, 2011.

22. Ganda K and Seibel MJ: Rapid biochemical response to denosumab in fibrous dysplasia of bone: Report of two cases. Osteoporos Int 25: 777-782, 2014.

This work is licensed under a Creative Commons Attribution-NonCommercial-NoDerivatives 4.0 International (CC BY-NC-ND 4.0) License. 